\title{
Trombocitopenia inmune primaria aguda presentación clínica y manejo en pacientes pediátricos, Hospital Mario Catarino Rivas.
}

\author{
Acute primary immune thrombocytopenia clinical presentation and management \\ in pediatric patients, Hospital Mario Catarino Rivas.
}

\section{Alejandra María Aldana Raudales*, Ana Aldana Raudales**, Allan Mauricio Aldana Raudales**, \\ Roxana Martínez****.}

\section{RESUMEN}

Antecedentes: La trombocitopenia inmune primaria, conocida como púrpura trombocitopénica idiopática (PTI), es un trastorno autoinmune adquirido que afecta adultos y niños. Objetivo: Caracterizar los pacientes pediátricos con PTI aguda mediante su identificación y seguimiento en el Hospital Nacional Mario Catarino Rivas (HNMCR) entre 1 febrero del 2016 al 31 enero del 2017. Pacientes y Métodos: Estudio cuantitativo, diseño no experimental longitudinal - prospectivo, alcance descriptivo. La población es todo paciente menor de 18 años de edad, que acudió al HNMCR con PTI aguda. La muestra 47 pacientes. Excluidos pacientes previamente diagnosticados con PTI, con segundos episodios o recaídas, con patologías sobre agregadas. Información recopilada mediante un instrumento tipo encuesta: situación demográfica, área geográfica y revisión del expediente clínico, los datos fueron analizados con el programa EPIINFO y EXCEL. Resultados: En cuanto al grupo etario el $56 \%$ fueron menores de cinco años de edad, la distribución por sexos hubo predominio del sexo masculino. $81 \%$ de los niños fueron hospitalizados, el resto recibió

\footnotetext{
* Residente de tercer año, Posgrado de Pediatría, Universidad Nacional Autónoma de Honduras en el Valle de Sula (UNAH-VS).

**Médico General.

*** Estudiante de séptimo año, Escuela Universitaria de las Ciencias de la Salud (EUCS, UNAH-VS).

*** Hematooncóloga Hospital Nacional Dr. Mario Catarino Rivas. Dirigir correspondencia a: alealdana286@hotmail.com

Recibido: 20 de diciembre 2016 Aprobado 08 de febrero 2017
}

manejo ambulatorio; sin embargo en el 100\% se inició alguna terapia con resolución de la trombocitopenia a los 3 meses. Luego del seguimiento, el diagnóstico final de los casos captados fue PTI aguda 58\%(n=27), evolucionaron a la cronicidad $20 \%(n=9)$, el $22 \%(n=10)$ abandonaron seguimiento. Conclusiones: La mayoría de los pacientes con PTI son admitidos al hospital, aunque su conteo de plaquetas no los ponga en riesgo claro de hemorragias espontáneas, por no haber guías de manejo en nuestro hospital.

\section{PALABRAS CLAVE}

Purpura, Immune, Trombocitopenia.

\section{ABSTRACT}

Background: Primary immune thrombocytopenia, known as idiopathic thrombocytopenic purpura (ITP), is an acquired autoimmune disorder that affects adults and children. Objective: to characterize pediatric patients with acute ITP by its identification and follow-up in the Hospital Nacional Mario Catarino Rivas (HNMCR) between February 1, 2016 and January 31, 2017. Patients and Methods: Quantitative study, non-experimental longitudinal - prospective design. Scope of the descriptive investigation. The population is all patients under 18 years of age, who attended the HNMCR with signs and symptoms suggestive of acute ITP. The sample shows 47 patients. Excluded patients previously diagnosed with ITP, with second episodes or relapses, with 
pathologies over aggregates. Information collected through a survey-type instrument: demographic situation, geographical area and review of the clinical file, the data was ana lyzed with the EPIINFO and EXCEL program. Results: As for the age group, 56\% were under five years of age, the sex distribution was predominantly male. $81 \%$ of the children were hospitalized, the rest received outpatient treatment; but in $100 \%$ some therapy was initiated. After the follow-up, the final diagnosis of the cases captured was acute ITP 58\% ( $n=27)$, they evolved to chronicity $20 \%(n=9), 22 \%(n=10)$ abandoned follow-up. Conclusions: The majority of patients with ITP are admitted to the hospital, although their platelet count does not put them at clear risk of spontaneous bleeding, because there are no management guidelines in our hospital.

\section{KEYWORDS}

Purpura, Immune, Thrombocytopenia.

\section{INTRODUCCIÓN}

La PTI es un trastorno autoinmune adquirido

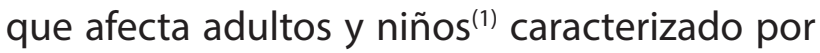
una destrucción incrementada de las plaquetas y se asocia con una producción deficiente de las mismas. ${ }^{(2)}$ Antes del 2009 se llamó «Púrpura Trombocitopénica Idiopática», con su acrónimo PTI. A partir de ese año, se prefirió reemplazar «idiopática» con «inmune» para enfatizar el mecanismo inmunológico de la enfermedad y «primaria» (en oposición a idiopática) al indicar la ausencia de cualquier otra causa obvia y/o subyacente. Se retiró el término "púrpura» ya que se consideró inadecuado, porque los síntomas de sangrado son ausentes o mínimos en una gran proporción de casos. ${ }^{(3)}$

La incidencia se calcula en 4 - 10 casos por 100,000 niños por año, con predominio de edad entre los dos y ocho años. ${ }^{(4)}$ Constituye la causa más frecuente de trombocitopenia en el niño, además, es la afección hemorrágica adquirida y autolimitada más frecuente en la infancia. Alrededor de un 3\% de los pacientes con PTI tienen manifestaciones clínicas de importancia. Recuentos plaquetarios menores a 10,000/uL, se correlacionan con sangrados más severos. La media de edad de presentación es 5.7 años, en un rango que va entre 1 y 10 años en un $70 \%$ de los casos y sin diferencia de sexo. ${ }^{(5)}$ El mecanismo fisiopatológico central es la pérdida de auto tolerancia a los propios antígenos localizados en la superficie de las plaquetas y los megacariocitos, lo que conlleva a la producción de autoanticuerpos principalmente de tipo IgG específicos contra las glicoproteínas Ib, Ilb, IIla. ${ }^{(6)}$

Se clasifica en aguda, crónica y recurrente. La PTI aguda: representa el 85\%, su duración es generalmente de un mes, pero puede durar hasta seis meses. La PTI crónica: tiene una duración mayor de seis meses. Hasta $20 \%$ de ellos consiguen remisión espontánea, la que se puede demorar hasta diez años. PTI recurrente o intermitente: representa menos del 5\%, la recurrencia consiste en que los pacientes tienen intervalos libres de la enfermedad, tanto en los síntomas como en las alteraciones de las pruebas de laboratorio, de duración promedio de tres meses. ${ }^{(7)}$

Entre las manifestaciones clínicas se encuentran epistaxis, equimosis, hemorragia gastrointestinal, hematuria o menorragia. La hemorragia intracraneal es infrecuente afectando aproximadamente $0,1-0,5 \%$ de los casos. Los factores de riesgo de hemorragia intracraneal en niños con trombocitopenia severa incluyen trauma craneal y uso concomitante de medicamentos que afecte a la función plaquetaria. ${ }^{(8)}$

Entonces podemos decir que la sintomatología 
de la trombocitopenia depende de la severidad de la misma. Así puede ir desde asintomático hasta sangrados severos que ponen en riesgo la vida de la persona. ${ }^{(9)}$

El diagnóstico de PTI se establece por exclusión sistemática de otras causas de trombocitopenia. El abordaje inicial se basa en la historia clínica, la exploración física, el hemograma y el exámen de la extensión de sangre periférica. Se deben evaluar las características de la hemorragia, si es cutánea o en mucosas, su gravedad, extensión y tiempo de evolución. Han de identificarse condiciones concomitantes que aumenten el riesgo hemorrágico. ${ }^{(10)}$ Para el diagnóstico de PTI deberán estar presentes los cuatro requisitos siguientes: Trombocitopenia (recuento plaquetario menor de 100,000/ $\mu \mathrm{l}$ ), ausencia de enfermedad infecciosa aguda concomitante, ausencia de patología sistémica de base, y megacariocitos normales o aumentados en médula ósea.

Frente a una situación de hemorragias con riesgo inmediato para la vida, se deben tomar las siguientes medidas en forma simultánea: lgG-IV: $1 \mathrm{~g} / \mathrm{kg} /$ día, por 1-2 días consecutivos, metilprednisolona: 30 mg/kg/día, intravenosa, por 2-3 días consecutivos, transfusión continúa de concentrado de plaquetas a 1 UI/hora, esplenectomía de urgencia y/o cirugía en el sitio de sangrado, si es necesaria y factible (sistema nervioso central, abdomen).. ${ }^{(11)}$

El objetivo fue caracterizar los pacientes pediátricos con Trombocitopenia Inmune Primaria aguda mediante su identificación y seguimiento en el Hospital Nacional Dr. Mario Catarino Rivas durante el periodo comprendido entre 1 febrero del 2016 al 31 enero del 2017.

\section{PACIENTES Y MÉTODOS}

Se realizó un estudio de enfoque cuantitativo, diseño no experimental longitudinal - prospectivo, con alcance descriptivo, en el HNMCR durante el periodo comprendido entre 1 febrero de 2016 al 31 enero de 2017. La población es todo paciente menor de 18 años de edad, que acudió al HNMCR con diagnóstico de PTI.

Durante el período del estudio 76 pacientes con PTI fueron atendidos en el área de emergencia y en consulta externa de Hemato-Oncología Pediátrica del HNMCR, de los cuales 47 cumplían con los criterios de inclusión: paciente pediátrico, con diagnóstico de PTI AGUDA independientemente del sexo, con conteo plaquetario confirmado en el hospital menor a 100,000 , cuyos padres o encargados, aceptaran participar y firmaron el consentimiento informado. Los otros 29 pacientes no fueron PTI agudas, consultaron con recaídas de PTI (n: 5/29) o bien por presentar evolución crónica de su enfermedad (n: 9/29 (48\%), o por tener plaquetopenias secundarias a otras enfermedades sistémicas (n: 15/29 (52\%). Información recopilada mediante un instrumento tipo encuesta con ítems sobre situación demográfica, área geográfica y revisión del expediente clínico. La información fue ingresada en una base de datos (Programa Epilnfo 7.2 para Windows 3.5, CDC, Atlanta, GA, EUA). Los resultados se presentaron como proporción, frecuencias, porcentajes, medidas de tendencia central y dispersión (promedio y desviación estándar).

Aspectos Éticos: La base de datos y formularios fueron protegidos, teniendo acceso solamente el investigador del estudio. Para la recolección de los datos se contó con el consentimiento informado escrito de la madre, padre o encargado del paciente, a quien se le garantizó la confidencialidad de la información que brindó. Se garantizó el principio de respeto a la dignidad humana, beneficencia y justicia. 


\section{RESULTADOS}

Las características demográficas de la población atendida muestran una distribución equitativa en cuanto a los diferentes grupos de edad (ver tabla No. 1), encontrando 56\% (n: 26/47) menores de cinco años; con promedio de 6.4043 años ( \pm 4.8259$)$, moda de 1. La distribución por sexos no es equitativa en este estudio. Hay un franco predominio del sexo masculino esto se debe a que la mayor parte de pacientes que acudieron a consultas por plaquetopenia fueron hombres, 32 de los 47 pacientes que representa un $59 \%$ con una proporción hombre - mujer de 2.1:1.

Tabla No. 1: Distribución de pacientes pediátricos diagnosticados con PTI de acuerdo a características sociodemográficas; $\mathbf{n}=47$.

\begin{tabular}{lc}
\hline Características & $\mathbf{n ~ ( \% )}$ \\
\hline Edad ( años) & $13(28) \%$ \\
1 mes -2 años & $13(28 \%)$ \\
$3-5$ años & $12(25 \%)$ \\
$6-11$ años & $9(19 \%)$ \\
$12-18$ años & \\
Sexo & $15(32 \%)$ \\
Mujer & $32(68 \%)$ \\
Hombre & \\
Departamento & $20(43 \%)$ \\
Cortés & $6(13 \%)$ \\
Yoro & $6(13 \%)$ \\
Colón & $4(8 \%)$ \\
Atlántida & $4(8 \%)$ \\
Copán & $3(7 \%)$ \\
Lempira & $2(4 \%)$ \\
Santa Bárbara & $1(2 \%)$ \\
La Paz & $1(2 \%)$ \\
Olancho & \\
Situación Demográfica & \\
Urbana & $26(55 \%)$ \\
Rural & $21(45 \%)$ \\
\hline
\end{tabular}

Fuente: PTI Aguda, HNMCR, ene 2016 - jun 2017
De acuerdo a la información brindada en las encuestas y expedientes clínicos, el $72 \%$ (n: 34/47) de los pacientes con PTI consultaron referidos de Centros de Salud, Hospitales de área, o bien por médicos particulares u hospitales privados. El sitio de captación de estas referencias sigue siendo la Emergencia de Pediatría, y pocos (n: 13/47) fueron evaluados de inicio en la consulta de Hemato-Oncología.

Otro dato relevante es el antecedente de infección en las semanas previas al inicio de la púrpura. Once de 47 padres reconocieron una infección (23\%). Entre las infecciones descritas se encuentran: procesos respiratorios (n: $7 / 11)$, gastrointestinales ( $n: 2 / 11)$, otros: otitis/dengue (n: $2 / 11$ ).

Las manifestaciones clínicas comunes fueron las cutáneas con equimosis ( $\mathrm{n}: 22 / 47$ ) y petequias (n: 11/47); seguidas por los sangrados nasales ( $\mathrm{n}: 8 / 47$ ) y con menor frecuencia gingivorragias y menorragias. El $11 \%$ (n: 5) de los pacientes con trombocitopenia inmune primaria presentaron trombocitopenia aislada.

En cuanto al recuento plaquetario inicial de los 47 pacientes, el promedio de $29,753 / \mu \mathrm{l}$. (DE \pm $25,622)$ y moda de $7,000 / \mu \mathrm{l}$. Del total de paciente, el 55\% (n: 26/47) al ser diagnosticados con PTI tenían un recuento inicial de plaquetas $<30,000 / \mu$ l.

En cuanto a las decisiones terapéuticas, $81 \%$ ( $\mathrm{n}$ : 38/47) de los niños fueron hospitalizados, el resto recibió manejo ambulatorio; pero en el $100 \%$ se inició alguna terapia; es decir, no hubo manejo expectante en estos pacientes.

En la Tabla No. 2 se presenta la distribución de los pacientes en el seguimiento y tiempo de resolución del cuadro de PTI. Un paciente fue referido con su médico local para seguimiento, 
por lo que no se tienen los controles de hemograma posterior al alta en el expediente. Los otros pacientes continuaron con evaluaciones posteriores, (Ver Tabla No. 2).

De los 46 pacientes que tuvieron seguimiento, el 76\% (n: 35/46) tuvieron hasta 6 controles, con una moda de $1-3$ controles y el $57 \%$ ( $n$ : 26/47) continuaron su tratamiento con esteroides orales. Del total de pacientes estudiados, el 58\% (n: 27/46) resolvieron en menos de 6 meses, el 22\% (n: 10/46) abandonaron el seguimiento, y $20 \%$ (n: 9/46) continúan en control. Ningún paciente falleció a consecuencia de la enfermedad. En el gráfico No. 1 se graficó la tendencia de respuesta al tratamiento después de iniciada la terapia en la población inicial y en los pacientes con plaquetas $<30,000 / \mu \mathrm{l}$.

Tabla No. 2: Distribución de pacientes pediátricos diagnosticados con PTI de acuerdo a su evaluación y resolución; $n=47$.

\begin{tabular}{lc}
\hline \multicolumn{1}{c}{ Características } & $\mathbf{n}(\%)$ \\
\hline Evaluaciones Posteriores & \\
SI & $46(98 \%)$ \\
NO & $1(2 \%)$ \\
Resolución de PTI (n: 46) & \\
1-2 meses & $14(30 \%)$ \\
3-4 meses & $8(17 \%)$ \\
5-6 meses & $5(11 \%)$ \\
Abandono de Seguimiento & $10(22 \%)$ \\
PTI Crónica & $9(20 \%)$ \\
\hline
\end{tabular}

Fuente: PTI Aguda, HNMCR, ene 2016 - jun 2017

Los pacientes a los que se perdió el seguimiento (n: 10/47, 22\%), resolvieron la PTI (conteo mayor de 100,00 plaquetas) en menos de tres meses los diez casos. Estos pacientes a los que hubo pérdida de seguimiento procedían de zonas lejanas al hospital (n: 8/10, 80\%) como
Copán, Lempira, Olancho, Yoro y Atlántida. En su último control $60 \%$ (6/10) tenían más de $150,000 / \mu l$ plaquetas.

Las características de la población que evolucionó a la cronicidad 56\% (n: 5/9) eran hombres, predominantemente preescolares, y debutaron con plaquetopenia menor a $30,000 / \mu \mathrm{l}$ (n: 7/9, 78\%).

Con respecto a los pacientes hospitalizados el $61 \%$ (n: 23/38) se debió a conteo plaquetario $<30,000 / \mu \mathrm{l}$, el $18 \%$ (n: $7 / 38$ ) por sangrado activo y el $21 \%$ (n: $8 / 38$ ) por otras causas como ser: antecedente de trauma, coinfección, escases de recursos económicos para movilización, procedencia lejana. (Ver tabla No. 3).

Tabla No. 3: Características de pacientes pediátricos menores de 18 años de edad diagnosticados con PTI que requirieron hospitalización; $\mathbf{n = 3 8}$.

\begin{tabular}{lr}
\hline Características & n (\%) \\
\hline Causa de Hospitalización & \\
Plaquetas $<30,000 / \mu l a$ & $23(61 \%)$ \\
Sangrado Activo & $7(18 \%)$ \\
Otros & $8(21 \%)$
\end{tabular}

Plaquetas iniciales

$\begin{array}{ll}30,000 / \mu \mathrm{la} & 23(61 \%) \\ 30,000-100,000 / \mu \mathrm{la} & 15(39 \%)\end{array}$

Terapia Inicial Instaurada SI

$38(100 \%)$

Tratamiento Inicial

ESTEROIDES IV

$21(55 \%)$

ESTEROIDES ORALES $\quad 9(24 \%)$

INMUNOGLOBULINA IV $3(8 \%)$

E. IV +G IV $3(8 \%)$

E. IV + PLT 2 $2(5 \%)$

Fuente: PTI Aguda, HNMCR, ene 2016 - jun 2017 
El 100\% contaron con terapia inicial, el 55\% (n: 21/38) la iniciaron con esteroides intravenosos, el resto con esteroides orales ( $n$ : 9/38), inmuno- globulina (n: 3/38), esteroides IV mas Inmunoglobulina ( $\mathrm{n}: 3 / 38$ ) y esteroides IV más plaquetas (n: 2/38).

\section{Gráfico No. 1: Variaciones mensual del recuento plaquetario}
A. En la población total
B. En los pacientes con PTI y Plaquetas menor a $<30,000 / \mu \mathrm{l}$.

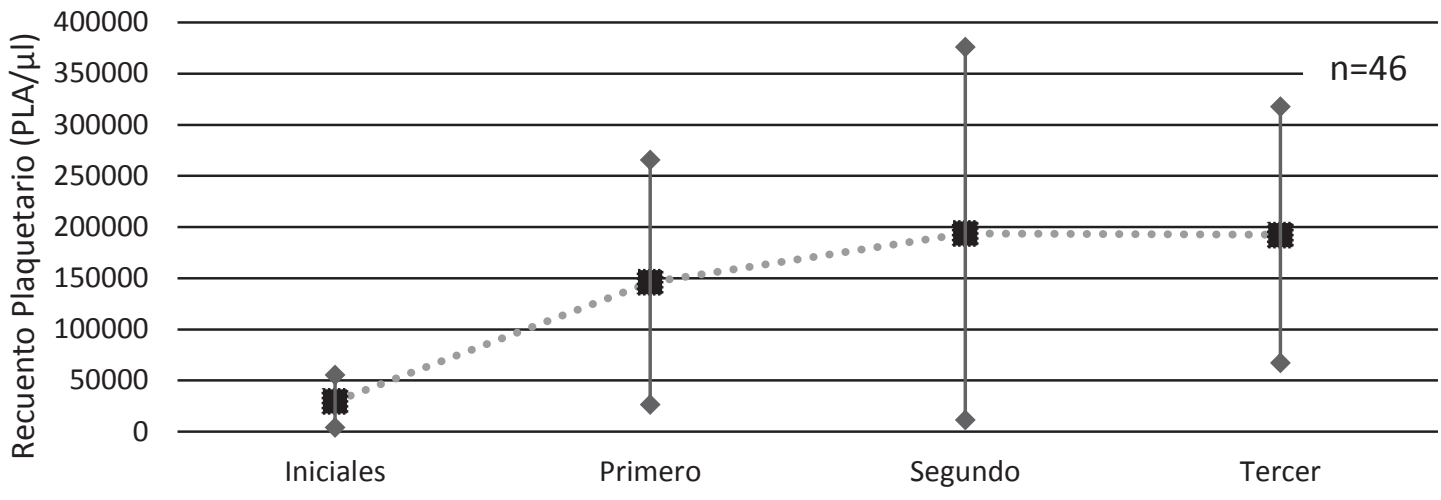

A.

Control mensual

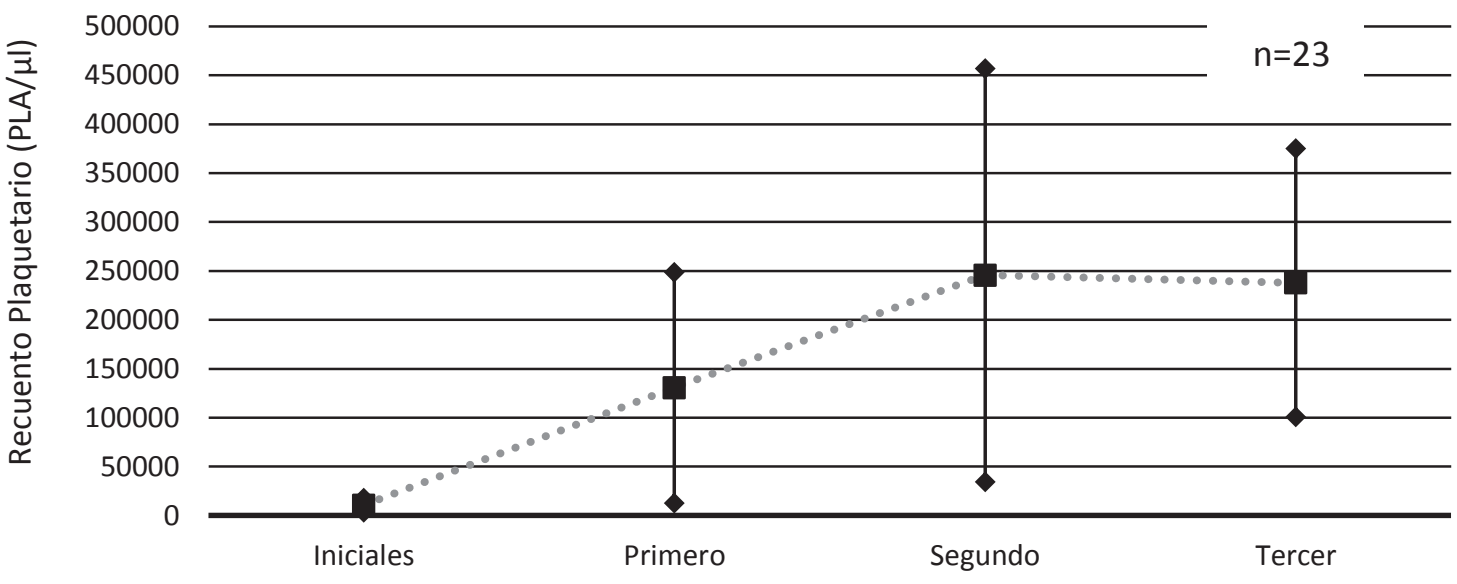

B.

Control mensual

Fuente: PTI Aguda, HNMCR, ene 2016 - jun 2017

\section{DISCUSIÓN}

La PTI es una causa frecuente de consulta pediátrica, y en nuestro caso pudimos detectar 76 casos en un año. Aunque el $72 \%$ pacientes fueron referidos, debe tomarse en cuenta que el $28 \%$ consultaron espontáneamente en la Emergencia del hospital. Esto indica que la presencia de hemorragias en piel y/o mucosas es un signo de alarma para los padres de familia, quizá en parte debido a la promoción del diagnóstico temprano de cáncer, que incluye en sus signos las equimosis y epistaxis.

Según las guías clínicas de países vecinos como El Salvador la mayoría de niños que desarrollan PTI son tratados ambulatoriamente con monitoreo de los niveles de plaquetas y las manifestaciones de sangrado. Los pacientes que requieren intervención farmacológica con inmunoglobulina o altas dosis de esteroides 
intravenosos son usualmente hospitalizados por uno o dos días, evaluados y manejados por el especialista en hospital de tercer nivel. ${ }^{(12)} \mathrm{A}$ diferencia que, en nuestra institución, de los pacientes estudiados el $81 \%$ de los niños fueron hospitalizados, en parte debido a que no hay guías claras sobre cuando hospitalizar o no, y en parte porque tradicionalmente se admiten para completar estudios como frotis de sangre periférico, algunas pruebas virales, etc.; aunque estos podrían hacerse ambulatoriamente. El hospitalizar a todos estos niños aumenta significativamente los costos para la institución y también para la familia, y sobre todo satura los cupos que se requieren para pacientes más complicados.

La PTI no tiene predominio en ningún sexo, y por eso se vuelve difícil explicar la presencia de $68 \%$ varones. No parece haber un factor cultural en este caso, pues en otras patologías no aparece esta diferencia en el momento de consultar a emergencias. Quizá se deba en parte a que las niñas tienen juegos menos agresivos y por tanto menor riesgo de sufrir equimosis que alarmen a los padres.

En un estudio realizado de la base de datos de Child Health Corporation de América en Kansas, EEUU, la mayoría de los pacientes presentaron sólo manifestaciones cutáneas leves tales como hematomas y petequias. Un 3\% de niños reportados sufrieron graves sangrados, incluyendo epistaxis grave, hemorragia extensa de la piel y mucosas, y sangrado gastrointestinal. La rara, pero más temida complicación, fue una hemorragia intracraneal que ocurre en, el $1 \%$ de los niños afectados por PTI. ${ }^{(13)} \mathrm{Al}$ igual que en este estudio, las manifestaciones clínicas más comunes fueron las cutáneas: equimosis $47 \%$, petequias 24\%; seguidas de epistaxis en un $17 \%$ y con menor frecuencia gingivorragias y menorragias. Ninguno se manifestó con una hemorragia intracraneana.
Casi el $60 \%$ de los casos del HNMCR resolvieron antes de 6 meses, y hay que considerar que un $22 \%$ se perdieron de seguimiento antes de los seis meses, pero tenían conteos cercanos a 150,000 en los últimos controles que constan en sus expedientes, de modo que es muy probable que la causa del abandono haya sido la resolución del cuadro clínico. El 20\% evolucionó a la cronicidad. Estos datos son muy similares a los encontrados en la Pediatría Integral publicada en el 2012 donde recalcan que el seguimiento de los pacientes con PTI debe individualizarse en función de la estabilidad del recuento de plaquetas y de la modalidad terapéutica. La evolución de la PTI es variable e impredecible. La evolución habitual de la PTI aguda es la remisión espontánea en el 70-80\% de los casos en los 6 meses siguientes. Evoluciona a una PTI crónica aproximadamente el $20 \%$ de los niños que presentan PTI aguda. ${ }^{(14)}$

Dentro de los datos discrepantes en esta investigación con respecto a la literatura internacional, solo el $21 \%$ de los padres reconocieron una infección. Esto puede deberse a negligencia de los padres en notar un proceso infeccioso quizá leve, ya que al momento de consulta inicial la gran mayoría niega infecciones en las semanas previas, probablemente debido a que los horarios de trabajo no les han permitido notar procesos leves que no requirieron consulta médica y resolvieron de manera espontánea; pues es poco probable que nuestros niños tengan una fisiopatología diferente a la clásicamente descrita para la enfermedad.

De acuerdo a las recomendaciones de Journal of Medical Economics el tratamiento inicial para la PTI a menudo incluye corticosteroides, y la inmunoglobulina intravenosa (IVIG) y anti-D. ${ }^{(15)}$ En el HNMCR, los pacientes con plaquetas $<30,000 / \mu$ l recibieron como terapia inicial combinaciones de esteroides IV con 
plaquetas o inmunoglobulinas en un $8 \%$, con inmunoglobulinas el $12 \%$ y con pulsos de esteroides en un $80 \%$. En este estudio las conductas terapéuticas más usadas fueron en un $74 \%$ esteroides como monoterapia y en una mínima cantidad combinada con inmunoglobulina en un $7 \%$. Otras alternativas terapéuticas son excepcionalmente necesarias, excepto en los pacientes que evolucionan a cronicidad.

La mortalidad por PTI ha sido baja en un $0.1 \%$ siempre secundaria a hemorragia del $\mathrm{SNC}^{(7)}$ lo lo cual discrepa de este estudio ya que la mortalidad en pacientes con PTI fue nula y no se presentó ningún caso de hemorragia intracraneana.

En conclusión, la PTI es una causa frecuente de consulta pediátrica en nuestro país, y en nuestro hospital ocurre por referencia de las zonas de influencia, así como por consultas espontá- neas de los padres. No hay guías claras de manejo para el médico de urgencias, de modo que muchos de estos pacientes son admitidos al hospital, aunque sus conteos de plaquetas no los pongan en riesgo claro de hemorragias espontáneas.

Se recomienda que deban generarse guías claras de manejo para las diferentes patologías pediátricas, en especial para la $\mathrm{PTI}$, dada su frecuencia, de modo que los pediatras laborando en diferentes hospitales y centros de salud puedan manejarla apropiadamente sin necesidad de referir al paciente obligándole a recorrer horas de camino. Además, mantener disponibilidad de los medicamentos recomendados en las guías de manejo (Metilprednisolona, Inmunoglobulina IV, etc.) en los diferentes hospitales, de manera que puedan cumplirse oportunamente las recomendaciones terapéuticas.

\section{REFERENCIAS BIBLIOGRÁFICAS}

1. Roque. W, "Trombocitopenia inmune primaria refractaria: opciones terapéuticas", Revista Cubana de Hematología, Inmunología y Hemoterapia, Vol. 28, Núm. 4 (2012). http://www.revhematologia.sld.cu/index. php/hih/article/view/4/11.

2. Publicación de la Agrupación Mexicana para el Estudio de la Hematología, A.C. Marfil. L "Trombocitopenia inmunitaria primaria" Rev. Hematol Mex 2015; 16: Suplemento 1, s50

http://www.revistasmedicasmexicanas. com.mx/nieto/Hematologia/2015/suple mento1/ HemaSupl1small. pdf.

3. García. B y colaboradores "Trombocitope- nia inmune primaria", septiembre-Diciembre 2015 / Volumen 10, Número 3. p. 154-165. http://www.medigraphic.com/ pdfs/residente/rr-2015/rr153f.pdf.

4. Ochoa. W y otros, "Púrpura Trombocitopénica inmune en la infancia" 20 años de experiencia: 1985-2005. Estado CojedesVenezuela, Arch Venez Puer Ped v.71 n. 4 Caracas dic. 2008 http://www.scielo.org. ve/scielo.php?pid=S000406492008000 400002\&script=sci_arttext\&tlng=en.

5. Verrugo. P, "Guías clínicas para el manejo del paciente pediátrico con trombocitopenia inmune primaria "(PTI), Rev. chil. pediatr. vol.82 no.4 Santiago ago. 2011 
http://www.scielo.cl/pdf/rcp/v82n4/art10. pdf.

6. Acon. E, “Purpura Trombocitopénica Inmunitaria" Hemato- Inmunología, Revista Médica De Costa Rica Y Centroamérica LXXI (611) 509 - 514, 2014. http://www.binasss. sa.cr/revistas/rmcc/611/art25.pdf.

7. Posada. A, "Púrpura trombocitopénica autoinmune en niños y adolescentes", CCAP Año 2003 Módulo 2 - 39

https://scp. com.co/precop-old/precop_fi les/modulo_3_vin_3/precop_ano3_mod3 _purpura. pdf.

8. Fierros. A, "Púrpuras. Trombocitopenia inmune primaria" Centro de Salud Pisuerga. Valladolid, Pediatr Integral 2012; XVI(5): 399-412

http://www.pediatriaintegral.es/wp-content/uploads/2012/xvi05/05/Purpuras.pdf.

9. Sequeira. L, "PURPURA TROMBOCITOPENICA AUTOINMUNE" (Caso Clínico y Revisión Bibliográfica) REVISTA MEDICA DE COSTA RICA Y CENTROAMERICA LXV (582) 9-13; 2008 http://www.binasss.sa.cr/revistas/ rmcc/582/art3.pdf.

10. Sanz y otros, Diagnóstico, tratamiento y seguimiento de la trombocitopenia inmune primaria; Sociedad Española de Hematología y Hemoterapia y la Sociedad Española de Hematología y Oncología Pediátricas; Med Clin (Barc). 2012; 138(6):261.e1-261.e17;

http://www.elsevier.es/es-revista-medicina -clinica-2-articulo-diagnostico-tratamien to-seguimiento-trombocitopenia-inmune -90098208 .
11. Donato, Púrpura trombocitopénica inmunitaria. Guía de diagnóstico y tratamiento; Comité Nacional de Hematología; Arch Argent Pediatr 2010;108(2): 173-178 http://www.scielo.org.ar/scielo.php?pi $d=$ S032500752010000200021\&script= sci_arttext.

12. MINISTERIO DE SALUD DE EL SALVADOR, "Guías Clínicas de Pediatría", San Salvador, febrero de 2012. http://apps.who.int/medi cinedocs/documents/s19170es/s19170 es.pdf.

13. Kime. C, y colaboradores, "Patterns of Inpatient Care for Newly Diagnosed Immune Thrombocytopenia in US Children's Hospitals", PEDIATRICS Volume 131, Number 5, May 2013 http://pediatrics.aappublica tions.org/content/131/5/880.long.

14. Fierr. A, "Púrpuras. Trombocitopenia inmune primaria", Pediatr Integral 2012; XVI(5): 399-412

https://www.pediatriaintegral.es/wp-con tent/uploads/2012/xvi05/05/Purpuras.pdf.

15. Prina Z. Donga, Sara P. Bilir, Gregg Little, Tim Babinchak \& Julie Munakata "Comparative treatment-related adverse event cost burden in immune thrombocytopenic purpura", Journal of Medical Economics, 08 October 2017 DOI:10.1080/13696998. 2017.1370425 http://dx.doi.org/10.1080/ 13696998.2017 .1370425$. 